\title{
DIPLOME UNIVERSITAIRE de perfectionnement en PATHOLOGIES des ORGANES GENITAUX EXTERNES
}

\author{
Hôpital COCHIN - TARNIER \\ 89 rue d'Assas 75006 Paris
}

Organisateurs : Professeur J.P. ESCANDE, O. AYNAUD, N. DUPIN, JM CASANOVA, B. HUYNH

Conditions d'inscription : Ouvert aux Dermatologues - Gynécologues - Urologues - Médecins généralistes.

Enseignement : 7 sessions de 2 jours (vendredi, samedi) Début de l'enseignement en Mai 2002

Frais d'inscription : 846,15 euros, Un dossier est déposé au FAF-PM dans le cadre de la formation médicale continue.

Inscription pédagogique : dernier délai le 10042002

Avec la collaboration de Dermatologues, de Gynécologue-Obstétriciens, d'Urologues et d'Anatomo-pathologistes

Conseil scientifique : O. AYNAUD, N. DUPIN, J-M. CASANOVA, B. HUYNH

AMARENCO G. (Hôpital Rothchild)

ASSOUS M. (Hôpital Cochin - Port Royal)

BENKIMOUN JM (Paris)

BERGERON C. (Lab. Pasteur Cerba)

BOURGAULT-VILLADA I. (Hôpital A Paré)

BODMER C. (Hôpital Necker)

BOUTONNET B. (Hôpital Lorquin)

CALVEZ V. (Hôpital Pitié - Salpêtrière)

CARLOTTI A. (Hôpital Tarnier)

CHAPRON C. (Hôpital Cochin - Port Royal)

CORNUD F. (Paris)

DELMAS V. (Hôpital Bichat)

DENIS J. (Hôpital Léopold Bellan)

DINTRANS JR. (Paris)

DROUAULT Y. (Boulogne-Billancourt)

FAVRE M. (Institut Pasteur)

FOULOT H. (Hôpital Cochin - Port Royal)
FRANCK N. (Hôpital Tarnier)

GUILLEMOTONIA A. (Paris)

GORIN I. (Hôpital Tarnier)

GOUREVITCH D. (Collège de France)

JANIER M. (Hôpital Saint Joseph)

KEITA XJ. (Barreau de Créteil)

LORTAT-JACOB S. (Hôpital Necker)

MANDELBROT L. (Hôpital Bichat)

MOYAL-BARRACO M. (Paris)

PANIEL JB. (Hôpital Intercommunal Créteil)

PELISSE M. (Paris)

PLANTIER F. (Paris)

SPIRA A. (Hôpital Bicêtre)

TEILLAC F. (Hôpital Pitié - Salpêtrière)

VIGIER C. (Hôpital Cochin - Port Royal)

WALLACH D. (Hôpital Tarnier)

WETTERVALD E. (Saint Mandé)

Le programme comportera des cours théoriques portant sur

Histoire des pathologies génitales

Organes génitaux et l'endocrinologie

Explorations des organes génitaux

Dermatoses non infectieuses, allergologie

Lésions bactério-fongiques génitales

Tumeurs virales génitales

Lésions génitales et ano-périnéales
Tumeurs génitales

Algies génitales

Chirurgie plastiques

Pathologies génitales chez l'enfant et l'adolescent

Traumatisme, rite et coutume

Esthétisme des organes génitaux

Sexualité et dermatoses génitales

Pour tous renseignements complémentaires ou pour obtenir un dossier de pré-inscription et ce avant le 15/04/2002 merci d'écrire ou de laisser vos coordonnées à :

Melle CHAMPION Janick Hôpital Tarnier 89, rue d'Assas 75006 PARIS Tél : 0158411781 - Fax : 0158411675 janick.champion@cch.ap-hop-paris.fr

aynaud@aol.com 